Chapman University

Chapman University Digital Commons

Communication Faculty Articles and Research

School of Communication

$10-27-2018$

\title{
Meta-Analysis of Anger and Persuasion: An Empirical Integration of Four Models
}

Nathan Walter

Northwestern University

Riva Tukachinsky

Chapman University, tukachin@chapman.edu

Ayellet Pelled

University of Wisconsin-Madison

Robin Nabi

University of California, Santa Barbara

Follow this and additional works at: https://digitalcommons.chapman.edu/comm_articles

Part of the Other Communication Commons, Other Psychology Commons, Personality and $\underline{\text { Social Contexts Commons, and the Social Influence and Political Communication Commons }}$

\section{Recommended Citation}

Walter, N., Tukachinsky, R., Pelled, A., \& Nabi, R. (2018). Meta-analysis of anger and persuasion: An empirical integration of four models. Journal of Communication, jqy054. doi: 10.1093/joc/jqy054

This Article is brought to you for free and open access by the School of Communication at Chapman University Digital Commons. It has been accepted for inclusion in Communication Faculty Articles and Research by an authorized administrator of Chapman University Digital Commons. For more information, pleasecontactlaughtin@chapman.edu. 


\title{
Meta-Analysis of Anger and Persuasion: An Empirical Integration of Four Models
}

\section{Comments}

This is a pre-copy-editing, author-produced PDF of an article accepted for publication in Journal of Communication following peer review. The definitive publisher-authenticated version

Walter, N., Tukachinsky, R., Pelled, A., \& Nabi, R. (2018). Meta-analysis of anger and persuasion: An empirical integration of four models. Journal of Communication, jqy054. doi: 10.1093/joc/jqy054

is available online at: DOI: 10.1093/joc/jqy054

\author{
Copyright \\ Oxford University Press
}




\title{
Meta-Analysis of Anger and Persuasion: An Empirical Integration of Four Models
}

\begin{abstract}
Despite the increasing use of anger in persuasive messaging, such as political ads and health campaigns, very little is known about when and how anger affects persuasion. Building on theoretical propositions derived from four theoretical models that have addressed the link between anger and persuasion, the current meta-analysis $(k=55, N=6,805)$ finds a weak impact of anger on behavior $(r=.15, p=.04)$ and nonsignificant effects on attitudes $(r=-.03, p=.30)$ and intent $(r=.06, p=.13)$. Yet, a closer look reveals a more complicated reality where positive effects are identified with the presence of strong arguments, relevant anger, and the inclusion of efficacy appeals. Further, the study identifies an interplay between emotional intensity and argument strength, such that argument strength plays an important role only at lower levels of anger. The study concludes by integrating the results proposing three promising areas for future research into anger and persuasion.
\end{abstract}

Keywords: Anger; persuasion; meta-analysis; emotional appeals; models of persuasion 


\section{Meta-Analysis of Anger and Persuasion: An Empirical Integration of Four Models}

Anger is considered to be one of the basic human emotions that occur universally across cultures (Ekman, 1999; Tomkins, 1962; Panksepp, 1994). It is, at least in the U.S., one of the most frequently experienced emotions in everyday life (Averill, 1982; Lerner \& Tiedens, 2006; Schieman, 2010). Although often considered in relation to interpersonal conflict, anger also plays an important role in persuasive communication, routinely serving as an emotional appeal in health and political campaigns (e.g., Lee \& Cheng, 2010). Yet academic researchers and media practitioners voice conflicting views concerning the effectiveness of this approach. Some argue that anger can be used as a powerful tool for mobilizing audiences to take action, as is evident from anger-fueled political campaigns (e.g., Kühne \& Schemer, 2015). Conversely, others warn against a possible backlash of anger appeals, as negativity from the persuasive message can be transferred onto the message sender (e.g., Van Dijk, Van Kleef, Steinel, \& Van Beest, 2008). Moreover, though several theoretical models have attempted to theorize how anger operates in the context of persuasion, research directly and systematically testing these propositions is relatively scarce.

To fill this gap, we conducted a meta-analysis that synthesizes and evaluates past research on anger and persuasion. In particular, we focus on experimental designs that manipulated anger and assessed its impact on message-consistent attitudes, intentions, and behaviors. Such synthesis of research across disciplines and theoretical frameworks offers a more complete understanding of how anger can be harnessed as an effective persuasive messaging tool. We begin with a discussion of anger as a discrete emotion and theoretical perspectives defining its role in the persuasion process. Next, we identify and test theoreticallygrounded predictors of persuasive outcomes and process-related variables. 


\section{Anger and Models of Persuasive Influence}

Like all basic emotions, anger is characterized by a unique pattern of cognitive, physiological, motivational, and behavioral responses that serve important evolutionary functions by directing attention to potentially consequential stimuli and promoting adaptive responses to the environment (Izard \& Ackerman, 2000; Kemper, 1987). Specifically, anger is elicited in situations that are appraised as an "unwarranted obstruction of goal” (Dillard \& Nabi, 2006 p. 125), “demeaning offense against me and mine” (Lazarus, 1993 p. 13), or a normative violation and as such, it is considered a negative emotional state.

Anger can be elicited by communication messages that threaten one’s ability to obtain personal goals (Dillard \& Nabi, 2006) and emphasize injustice (Rottenberg, Ray, \& Gross, 2007). Such appeals to fairness can be found in political campaigns blaming outgroups (e.g., immigrants) for benefiting at the expense of the message recipient (Brugge, 1995); in health contexts, where others (e.g., tobacco companies) are portrayed as manipulative and working against audiences’ best interests (Dillard \& Nabi, 2006); and in commercial contexts, where companies may be portrayed as taking advantage of unsuspecting consumers.

Communication messages can also elicit anger through representations of others' anger expressions. Due to process of emotional priming, emotion contagion, and identification, viewers may experience anger as a consequence of exposure to the angry demeanor of the person they observe. The effect of exposure to images of angry faces on viewers' display of anger has been well documented in psychology research (e.g., Dimberg, Thunberg, \& Elmehed, 2000). Outside the lab setting, this approach is used in strategic communication by presenting an outraged spokesperson.

At present, several distinct models, derived from communication, political science, and 
psychology, theorize the persuasive influence of messages that evoke anger. First, the cognitive functional model (CFM, Nabi, 1999) integrates cognitive models of persuasion (e.g., Petty \& Cacioppo, 1986) and functional theories of emotion (e.g., Lazarus, 1991) positing that media messages can elicit discrete emotions through a process of cognitive appraisal. Within this framework, each emotion is presumed to encompass emotion-specific motivational goals, giving rise to unique patterns of message processing and behavioral tendencies. These, in turn, predict persuasive outcomes.

Second, from political science, the affective intelligence theory (AIT, Marcus, 2013) takes a similar approach by incorporating an early rendition of the behavioral motivation model (Gray, 1978), dual-decision making models, and functional theories of emotions, to explain the role of emotions in political behavior (MacKuen et al., 2010). The AIT postulates that three simultaneous goal-oriented appraisal processes activate two neural systems (dispositional and alarm systems) producing enthusiasm/depression, anger (aversion), or anxiety. In turn, the activated emotion triggers learned scripts or call for further scrutiny. In contrast to the cognitive theories of emotions on which the CFM is based, the AIT focuses on pre-conscious, automatic processing which represents "a low level affective reaction" that precedes the "full blown emotional states” (Clore \& Ortony, 2008, p. 638). Given this focus, unlike the CFM, the AIT does not consider the many information-processing moderators germane to cognitive theories of emotion and theories of persuasion. Further, unlike the CFM, which focuses on emotional responses generated by the persuasive message itself, the AIT has been applied to situations when emotions evoked by one message guide attention to and effects of subsequent messages.

Third, the anger activism model (AAM, Turner, 2007) also theorizes the effect of anger appeal messages. Like the CFM, the AAM is rooted in the cognitive appraisal perspective, 
though focuses exclusively on anger. The model articulates ways in which efficacy beliefs and anger intensity can interact to produce distinct persuasive outcomes. More specifically, the AAM posits that although anger is a mobilizing force, individuals must feel capable of doing something about the source of the situation in order for this emotion to be productive. Thus, to maximize effectiveness, persuasive messages should generate high levels of anger coupled with self-efficacy beliefs.

The appraisal tendency framework (ATF) is another model that arguably relates to this context in that it suggests that emotional states predispose individuals to cognitively appraise subsequent events using those emotion-consistent appraisal patterns. However, whereas the previous models are primarily interested in anger elicited by messages themselves, ATF examines the effect of prior emotional states on evaluations in subsequent, unrelated situations, neither of which may be message based (Lerner \& Keltner, 2000). Although the ATF applies more readily to contexts of judgment and evaluation rather than persuasion per-se (Kim \& Cameron, 2011), it does provide numerous insights into the interplay between emotions and decision-making.

Unfortunately, despite advances in theorization of anger and persuasion, relatively little empirical research has tested the propositions advanced by these theories. The current metaanalysis synthesizes the existing body of research on anger and persuasion and articulates the process of anger elicitation and effect in the context of persuasion. It is suggested here that although the CFM, AIT, AAM, and ATF vary in scope and offer unique perspectives on anger and persuasion, these models share key assumptions about the functions of emotions and complement each other by collectively considering a wider array of ways in which anger appeals may operate. The following sections articulate hypotheses derived from these models, which are 
then tested in a meta-analysis that focuses both on the direct effects of anger on persuasive outcomes, as well as the underlying mechanism that can either augment or attenuate these effects.

\section{Effects of Anger on Persuasion}

Collectively, the CFM, AIT, AAM, and ATF maintain that anger has the potential to facilitate persuasion. Indeed, individuals who experienced higher levels of anger in response to an anti-smoking campaign expressed greater interest in taking action to stop smoking and were more likely to discuss the ad with others (Ilakkuvan, Turner, Cantrell, Hair, \& Vallone, 2017). Similarly, research guided by the AIT found that in a national sample, experienced anger was a significant predictor of political participation, controlling for self-efficacy and demographic characteristics. Moreover, anger, but not fear, predicted political participation that requires substantial investment and commitment (e.g., attending a rally as opposed to wearing a button) (Valentino et al., 2011). However, some studies failed to find a significant direct relationship between anger and message-consistent attitudes and behaviors (e.g., Kim \& Niederdeppe, 2014; Mitchell, Brown \& Morris-Villagran, 2001).

In light of these conflicting outcomes, it is, therefore, imperative to first identify the overall effect of anger on persuasive outcomes (attitudes, behavioral intentions, and behaviors) before moving to explore the conditions that facilitate or hinder this effect:

RQ1: What is the average effect of anger manipulations on attitudes, intentions, and behaviors?

\section{Message Variables and Persuasive Effect}

Next, the meta-analysis considers various theory-driven, message-related moderators of anger's persuasive effect, including anger relevance, argument strength, and efficacy cues. 
Anger relevancy. In examining the persuasive effect of anger, it is critical to distinguish incidental anger, ambient anger, and anger generated by the message's core-theme. Incidental anger is defined as an emotional by-product of the primary emotion elicited by the message. For example, excessive use of a guilt appeal can inadvertently evoke anger. Such incidental anger responses might lead to reactance and ultimately result in message rejection (Rains, 2013). Given the focus of this meta-analysis on intentionally evoked anger, messages that evoke anger unintentionally or coincidentally are excluded from consideration.

Both ambient and message-relevant anger are intentionally evoked, but have different relationships with message content. Ambient anger is elicited by events prior to message exposure, for example by recalling emotional events or watching anger-evoking images unrelated to the persuasive message topic. Ambient anger creates an "angry state of mind” with which individuals approach a persuasive message that is not inherently anger-evoking. From an ATF standpoint, ambient anger creates an appraisal tendency of higher control and certainty, resulting in greater optimism and attribution of responsibility (Lerner \& Keltner, 2000). Consequently, ambient anger can reduce cooperation and empathy in subsequent decision making (Small \& Lerner, 2008; Yip \& Schweitzer, 2016), facilitate harsher judgments of others (Polman \& Ruttan, 2012), and blunt emotional responsiveness to subsequent stimuli (Lerner \& Tiedens, 2006). Moreover, anger can serve as a heuristic cue signaling displeasure with the message and tainting judgments of the message overall (Schwarz, Bless, \& Bohner, 1991). In light of these effects, ambient anger is unlikely to be conducive to subsequent persuasion attempts.

In contrast, message-relevant anger, namely, anger evoked by the core content of the message, is conceptualized as productively related to persuasion (Dillard \& Nabi, 2006; Turner, 
2010). Specifically, according to the CFM, message-relevant anger motivates one to follow the message’s call for action to remove the threat. Thus, if anger is elicited by the appropriate emotion-specific core-relational theme embedded in the message itself (i.e., "a barrier or an affront”; Nabi, 1999 p. 307), it can generate desired persuasive influence. Building on this theorization, it is hypothesized that:

H1: Message relevant anger will produce stronger persuasive effect on attitudes, intentions, and behaviors than ambient anger.

Anger, argument strength, and processing motivation. Despite being classified as "unpleasant” or "negative” on the hedonic dimension, anger involves an approach motivation whose primary function is to mobilize individuals to confront threats and change undesirable situations. Indeed, on a physiological level, anger is associated with shifting blood flow from the viscera to the muscles, preparing the body for action (Izard \& Ackerman, 2000) and by higher cortical left hemisphere prefrontal activity, which is also characteristic of positive approach emotions (Harmon-Jones \& Allen, 1998, Harmon-Jones \& Sigelman, 2001). Similarly, subjective experience of anger encompasses approach attributes such as determination and alertness (Harmon-Jones, Harmon-Jones, Abramson, \& Peterson, 2009).

Consequently, the CFM highlights anger's likely influence on engagement with and processing of persuasive messages. Specifically, the CFM argues that anger's approach motivation will translate into closer engagement with the message itself, leading to more careful, and perhaps more critical assessment of the information presented. Such engagement can be operationalized, for instance, as amount of time spent processing the message, as well as more elaborate and critical thought processes. For example, De Los Santos and Nabi (in press) found that news-story generated anger lead to more time spent on the news webpage than fear or 
neutral affect. Further, Nabi (2002) found that those angered about domestic terrorism read a related policy story more carefully than those experiencing fear on the same topic, and Zuwerink and Devine (1996) found that intensity of irritation in response to a message strongly correlated with the sheer amount of negative cognitions listed by study participants. Similarly, Moons and Mackie (2007) demonstrated that anger made study participants more likely to discriminate between weak and strong arguments (even among individuals low-in need for cognition) and less likely to rely on dispositional heuristics, compared to participants in neutral moods. If anger promotes greater scrutiny then, ability to elaborate notwithstanding, argument quality should have a critical impact on the persuasive outcome of the message. As summarized by Mitchell, Brown, Morris-Villagran, and Villagran (2001), when people in angry moods are exposed to weak persuasive messages, they exhibit more unfavorable attitudes, less intention to change, and less behavioral change, compared to those exposed to strong arguments.

Thus, we advance the following hypothesis:

H2: Anger manipulations will produce a stronger persuasive effect on attitudes, intentions, and behaviors, when the message presents strong versus weak arguments.

Assurance and efficacy. Anger mobilizes individuals to take immediate action to resolve the anger-evoking situation. Thus, both the CFM and AAM assert that it is critical for the message to provide a viable avenue to direct the activated anger into action. The CFM argues that anger's influence on persuasive outcomes relies on the message recipients’ expectations about whether the message will assist in achieving the emotion-induced goal (Nabi, 1999). Thus, messages that offer, or are anticipated to provide, recommendations that will satisfy the emotion-induced goals should have a persuasive advantage. 
The AAM specifically articulates the presence of self- and response-efficacy appeals as the moderating factor that enhances message recipients' motivation to take action consistent with the persuasive message (Turner, 2007). Whereas self- and response-efficacy assurances empower and mobilize for activism, the absence of efficacy information is counterproductive. Accordingly, Turner (2007) suggests a typology based on a combination of levels of anger and efficacy: (1) the high anger-high efficacy group is marked by positive attitudes regarding the topic and willingness to engage; (2) the low anger-high efficacy group feels empowered but is likely to discount the importance of the topic; (3) the high anger-low efficacy group might have positive attitudes toward the topic but less assurance that they can facilitate a change; (4) and the low anger-low efficacy group is disinterested. As predicted, in the context of antismoking advertisements, message-consistent cognitions were highest among the high anger-high selfefficacy groups and lowest among the low anger-low self-efficacy groups (Ilakkuvan, Turner, Cantrell, Hair, \& Vallone, 2017). Thus, building on notions of the CFM and AAM, we hypothesize that the effect of an anger manipulation will be moderated by message expectations and efficacy cues such that:

H3: Anger manipulations will produce stronger effects on attitudes, intentions, and behaviors, when reassurance cues -- (a) self-efficacy and/or (b) response-efficacy cues -- are present in the message.

\section{Persuasive Effect of Anger Intensity}

To gain a more nuanced understanding of the effect of anger on persuasion, it is important to look not simply at manipulated anger but specifically at the intensity of the anger aroused from those manipulations and its subsequent effects on message processing ability and persuasive outcomes. It has been suggested that only low or moderate levels of anger facilitate a 
constructive approach to problem solving (e.g., Lewis, Sullivan, Ramsay, \& Alessandri, 1992), whereas high levels of anger result in cognitive deficits, as is evident from the strong correlations between anger and impulsivity (Ramírez \& Andreu, 2006).

From a message processing standpoint, at very low levels of anger, message recipients may remain disengaged and unmotivated to take action (Turner, 2007). Yet, intense anger might impede ability to process messages as the limbic system takes over and overrides more thoughtful, regulated, cortical-level processes (Goleman, 1995). Thus, extreme anger is likely to subvert systematic processing and increase reliance on heuristic cues (Loewenstein \& Lerner, 2003) or lead to disengagement from the anger-evoking message (Small \& Lerner, 2005). Thus, we may expect anger intensity to demonstrate a curvilinear relationship with persuasive outcomes, in light of the influence of low anger on impeding processing motivation and high anger on impeding processing ability.

It is unclear, however, whether experimental manipulations could produce such powerful levels of anger such that study participants' judgments would be clouded. That is, even in studies demonstrating a successful, strong manipulation of anger, study participants may be unlikely to lose their ability to think rationally. Given the body of research may not contain examples of extreme anger arousal allowing for a solid test of a curvilinear relationship between anger arousal and persuasive outcome, we pose the following research question:

RQ2: Is there a linear or curvilinear relationship between the intensity of self-reported anger on attitudes, intentions, and behaviors?

Finally, keeping in mind that according to the CFM, a potential drop in persuasiveness at higher levels of induced anger is attributed to the taxing of cognitive resources, it stands to reason that argument strength would play a more significant role at lower or moderate levels of 
anger, as opposed to high levels of anger. In other words, if individuals are overwhelmed with anger, systematic processing might be impossible due to resources shortage. Under these conditions, argument strength will not emerge as a significant moderator. We therefore ask: RQ3: Is the effect of the intensity of self-reported anger on attitudes, intentions, and behaviors moderated by argument strength?

\section{Method}

\section{Selection of Studies}

Literature search. Studies included in this meta-analysis were obtained in three ways. First, relevant electronic databases were systematically searched for empirical reports that focused on anger and persuasion (e.g., Google Scholar, All Academic, JSTOR, Medline, ProQuest, PubMed, Communication and Mass Media Complete, Educational Resources Information Center). The search located journal publications, conference papers, book chapters, and doctoral dissertations from a wide range of adjacent disciplines. The specific terms (and their derivations) that were used to perform the search included: anger, emotional appeals, appraisal theory, discrete emotions, negative emotions, conflict, and retaliation. These were combined with: persuasion, attitudes, intention, and behavior. Second, we examined the reference lists for each publication to find potential studies that were not located by our search terms. Finally, we contacted 10 leading scholars in the field of emotional appeals (i.e., those known for their long-standing research programs on emotion and persuasion and those whose work appeared repeatedly in our corpus) and asked them to identify omissions in our study corpus, as well as to share their unpublished results from relevant studies. Seven scholars replied, sharing 10 studies (seven published and three unpublished), four of which were later included in the dataset.

Inclusion criteria. To be included in the meta-analysis, studies had to meet the 
following four criteria. First, each study had to include a comparison between a condition where anger was purposefully induced and a neutral (non-emotional) equivalent condition. Second, studies had to measure the effects of anger (whether message relevant or ambient) on attitudes, intentions, or behavior. Third, studies had to report on quantitative outcomes. Finally, studies had to report appropriate statistics (e.g., $t$ values, means, standard deviations, counts, frequencies, zero-order correlations, or exact $p$ values) for calculating an effect size. In cases where the original report did not include sufficient data $(k=3)$, corresponding authors were contacted and the relevant data were successfully obtained. After the screening process, 36 research reports that documented results of 55 separate studies were included in the meta-analysis ( $16 \%$ unpublished), with a total sample size of 6,805 (see appendix A for a complete outline of the search strategy). The persuasive potential of anger was examined in a range of contexts, including politics $(k=11)$, negotiation $(k=11)$, health $(k=9)$, education $(k=7)$, crime $(k=4)$ marketing $(k=2)$, work $(k=2)$, and other $(k=9)$.

\section{Variable Coding}

Anger intensity. Overall, 28 studies employed a manipulation check to ensure that participants in the anger condition felt angrier compared to their counterparts in the neutral condition. In these studies, anger intensity was calculated as the standard difference in means (Cohen's $d$ ) of experienced anger between the anger condition and the neutral condition $(M=$ $0.99, S D=0.81)$. Then, for the purpose of consistency with other meta-analyses in the area of emotional appeals, Cohen's $d$ was transformed to Pearson's $r$. It is important to note that our inclusion criteria did not require studies to assess experienced anger for multiple reasons. First, there were no significant differences between effect sizes of studies that included manipulation checks and those that did not, $Q(1)=1.71, p=.20$. Second, in some cases, studies that measured 
experienced anger and studies that did not used the same anger-inducing manipulations (e.g., facial expression stimuli sets); hence these studies had de facto the same independent variables. Third, in some publications that report on several research studies with the same manipulation (e.g., Villalobos \& Sirin, 2017), the success of the induction method was assessed only once and then used in other studies. Excluding those studies without manipulation checks would have necessitate the removal of the later studies, which would undermine the integrity of these analyses. Given that focusing exclusively on studies with manipulation checks would have required the exclusion of 27 ( $50 \%)$ studies from the dataset, many of which were comparable to included studies in design, and given the comparable effect sizes as noted above, the lack of manipulation check was not deemed a productive exclusion criterion.

Persuasion outcomes. All effect sizes for attitudes, intentions, and behaviors were calculated separately per sample. Based on an explication provided by Rokeach (1968), an attitude refers to an organization of several favorable or unfavorable beliefs around a specific object or situation (e.g., attitudes toward bone marrow exams in Mitchell et al., 2001). Behavioral intent was conceptualized as a plan to behave in a way consistent with the persuasive message (e.g., intent to get on a bone marrow registry in Mitchell et al., 2001) and behavior dealt with the measurement of actual conduct (e.g., getting on the bone marrow registry in Mitchell et al., 2001). Similar constructs such as stage of change were not considered persuasion-related outcomes, unless the original study explicitly gauged participants’ attitudes or an intent to behave in a way consistent with the persuasive message. Though most samples $(k=45)$ included only one relevant outcome (i.e., attitudes, intentions, or behaviors), some samples included two $(k=9)$, as well as all three $(k=1)$ outcomes.

Coding of moderators. The meta-analysis focuses on a series of moderators, including 
anger relevancy, argument strength, and inclusion of efficacy cues. Thus, studies were coded for whether the aroused anger was ambient to the persuasive context (e.g., recollection of an unrelated emotional experience prior to exposure to the message) or relevant to the persuasion context (e.g., part of the persuasive message) ( $k=23$ and $k=32$, respectively). Further, the 11 studies that directly manipulated argument strength were used to compare the effects of strong persuasive arguments (i.e., 11 subsamples) with weak persuasive arguments (i.e., 11 subsamples). In addition, for studies that provided sufficient information about the persuasive stimulus, appeals to response- and self-efficacy were coded as present or absent. Approximately one-third of studies included response-efficacy appeals (present: $k=14$; absent: $k=41$ ). Few studies included self-efficacy appeals (present: $k=6$; absent: $k=49$ ). It is important to note that virtually all studies that included appeals to self-efficacy also included appeals to response-efficacy (see appendix B for complete information on the studies in the meta-analysis).

\section{Inter-Coder Reliability}

Approximately 55\% of our final sample $(k=30)$ were coded by two independent coders to assess inter-coder reliability. Krippendorff's alpha was used to assess the level of agreement between the coders and the reliability coefficient was at or above .81 for all variables, with the lowest reliabilities recorded for the variable of topic $(\alpha=.81)$ and self-efficacy $(\alpha=.84)$. These reliability levels indicate a satisfactory level of agreement and are actually compatible with other meta-analyses in the area of emotional appeals, including anger (minimum agreement was .80 in Tannenbaum, et al., 2015) and humor (minimum agreement was .79 in Walter, Cody, Xu, \&

Murphy, in press). Disagreements were resolved through discussion between the authors.

\section{Data Analysis}

Correlation coefficients $(r)$ were calculated using the statistical package Comprehensive 
Meta-Analysis (v.3; Borenstein, Hedges, Higgins, \& Rothstein, 2005). The study reports on uncorrected effect sizes based on random-effects assumptions (Hedges \& Vevea, 1998). Main analyses were done separately for each outcome variable, resulting in three meta-analyses that are independent of one another (for an example of this approach see Noar, Piece, and Black, 2010). Before proceeding with the moderation analysis in CMA, heterogeneity tests were conducted separately for each outcome using $Q$ statistics (Higgins \& Thompson, 2002). The research question concerning continuous variables (i.e., $R Q 2$ ) was tested with a meta-regression. Further, considering the fact that argument strength was coded at the subsample level (within studies) and experienced anger at the study level, to examine the interplay between experienced anger and argument strength (i.e., $R Q 3$ ), we first divided the sample of studies into low-moderate intensity and moderate-high intensity based on a median-split of experienced anger (Cohen's $d$ Med $=$ 0.64). We then conducted two separate moderation analyses of argument strength for each level of experienced anger.

\section{Results}

\section{Effects of Anger Elicitation}

Before testing the effects of anger on persuasion, the analysis examined whether studies were successful in eliciting experienced anger. Across 28 individual studies ${ }^{1}$ there was a positive, moderate-level, and significant effect of anger manipulation on experienced level of anger $(r=.42,95 \%$ CI $[.30, .53], p=.001)$, with significant heterogeneity in effect sizes $Q(27)=$ 591.95, $I^{2}=95.44 \%, p=.001$ (see appendix C for a forest plot of effect sizes with 95\% CI).

\section{Effects of Anger on Persuasion}

RQ1 asked about the overall effect of anger inductions on persuasive outcomes.

Interestingly, the only significant effect of anger was on behavior $(r=.15,95 \%$ CI $[.01, .28], p=$ 
$.04, k=13)$ and nonsignificant effects were recorded for attitudes $(r=-.03,95 \%$ CI [-.09, .03], $p$ $=.30, k=36)$ and intent $(r=.06,95 \%$ CI [-.02, .14], $p=.13, k=17)$. Keeping in mind the relative homogeneity of effect sizes for studies that assessed intentions $\left(Q(12)=23.38, I^{2}=\right.$ $48.67 \%, p=.09)$ and behavioral $\left(Q(16)=80.32, I^{2}=51.13 \%, p=.08\right)$ outcomes, the moderation analysis focuses on the interplay between anger and attitudes $\left(Q(35)=155.92, I^{2}=77.55 \%, p=\right.$ $.0005)^{2}$. Table 1 summarizes heterogeneity statistics and anger effects by relevant outcome.

\section{--- Table 1 ---}

\section{Moderators of Anger Manipulation Effect}

$\mathrm{H} 1$ predicted that message relevant anger would produce stronger persuasive effect than ambient anger. Anger's relevance to the persuasive outcome exerted significant influence on attitude-related effect sizes $(Q(1)=3.86, p=.04)$. A slight, though non-significant positive relationship was identified when anger was related to the persuasive context $(r=.03$, 95\% CI [$.07, .13], p=.54, k=32$ ), whereas ambient anger resulted in a significant and negative impact on attitudes $(r=-.09,95 \%$ CI [-.15, -.02], $p=.01, k=32)$.

It was next hypothesized that argument strength would interact with anger relevancy. As predicted by H2, argument strength significantly moderated $(Q(1)=5.38, p=.02)$ the effect of anger manipulations on attitudes such that messages that presented strong arguments resulted in significant, positive effect on attitudes ( $r=.09,95 \%$ CI [.02, .15], $p=.012, k=11$ ), whereas those with weak arguments indicated a negative, non-significant relationship ( $r=-.05$, 95\% CI [$.15, .05], p=.30, k=11)$.

To examine the moderating role played by message assurance (H3), we conducted a Qtest that compared the studies that included appeals to self-efficacy and/or response-efficacy with those that did not include such appeals. As expected $[Q(1)=6.04, p=.01]$, a positive, near 
significant effect of anger manipulations on attitudes was found for studies that included selfefficacy cues in the message ( $r=.08,95 \%$ CI $[-.01, .17], p=.06, k=6)$, whereas those that did not had a significant negative influence on attitudes $(r=-.06,95 \%$ CI [-.12, -.01], $p=.01, k=$ 49). Likewise, consistent with the hypothesis $[Q(1)=7.36, p=.007]$, messages that contained response-efficacy information had a significant and positive effect on attitudes $(r=.07,95 \%$ CI [.01, .13], $p=.04, k=14)$, and those that did not resulted in a significant negative effect on attitudes $(r=-.08,95 \%$ CI [-.15, -.01], $p=.04, k=41)$.

RQ2 inquired about a potential linear or curvilinear relationship between the intensity of self-reported anger and persuasion. This question was examined via a meta-regression by including the linear and quadratic terms of experienced anger as continuous predictors of effects on attitudes. Interestingly, based on the meta-regression $[Q(2)=19.58, p=.001 ; k=12]$, the effect of anger intensity on attitudes was curvilinear; linear term of experienced anger: $b=.31$, $S E=.43, p=.48$; quadratic term of experienced anger: $b=-.49, S E=.18, p=.01$. As the plotted regression line demonstrates (see Figure 1), positive, albeit weak, effects on attitudes occur at low to moderate-levels of anger intensity $(d<.40)$ and they gradually decrease at higher-levels of anger intensity $(d>.40)$, resulting in negative effects.

\section{--- Figure 1 ---}

Further, with respect to RQ3, the moderation of argument strength on anger-based attitudes was observed at low-moderate levels of anger intensity $[Q(1)=3.63, p=.04]$ but not at moderate-high levels $[Q(1)=0.14, p=.71]$. In particular, when anger was experienced at lowmoderate levels, strong arguments were more persuasive $(r=.10,95 \%$ CI $[.03, .16], p=.007, k$ $=6$ ), than weak arguments ( $r=-.04,95 \%$ CI [-.15, .08], $p=.54, k=6)$. Conversely, when anger was experienced at moderate-high levels, the relative strength of the persuasive message did not 
have a significant impact for either weak arguments, ( $r=.06,95 \%$ CI [-.18, .29], $p=.64, k=5)$ or stronger arguments $(r=-.01,95 \%$ CI $[-.28, .26], p=.93, k=5)$. Figure 2 presents the plotted interaction between anger intensity and argument strength.

--- Figure 2 ---

\section{Publication Bias}

A common method to detect a publication bias uses Kendall's Tau to estimate the relationship between the standardized effects and their variance (Begg \& Mazumdar, 1994). According to this estimate, there was no significant relationship between standardized effects and their variance (Tau $=.11, p=.23$ ), indicating no support for a file drawer problem. An alternative approach was introduced by Duval and Tweedie (2000). According to the Trim and Fill procedure, a) smaller studies causing plot asymmetry are removed, b) “true” averaged effect is estimated, and c) the center of the plot is filled with the omitted studies. Based on the trim and fill assessment in comprehensive meta-analysis, six studies had to be trimmed, resulting in a slightly stronger average effect of anger on persuasion $(r=.09,95 \%$ CI $[.02, .15])$ than the one observed in the data. With that in mind, these results should be interpreted with caution, as other evidence point to an opposite conclusion. Namely, when directly comparing published studies ( $k$ $=46$ ) with unpublished studies $(k=9)$ within our sample, the analysis records a significant difference $[Q(1)=6.01, p=.014]$, indicating that unpublished studies tend to record weaker effects $(r=-.05$, 95\% CI [-.11, .01], compared to published studies ( $r=.06,95 \%$ CI [-.01, .13].

\section{Discussion}

Despite the increasing use of anger in persuasive messaging, we know very little about the theoretical process underlying such effects. Focusing on message relevant versus ambient anger's effects on the processing of persuasive messages, this meta-analysis tests theoretical 
propositions derived from several models of emotions and persuasion. Although, on average, anger has a small and non-significant effect on persuasion, substantial variation across studies was uncovered for attitudinal outcomes. Specifically, more positive effects were identified with the presence of message-relevant anger, strong arguments, and the inclusion of efficacy appeals. Moreover, the analyses revealed an inverse U-shaped relationship between the intensity of anger and attitude-related outcomes, such that moderate-high levels of anger result in lower persuasive effects. Finally, whereas argument strength played a critical role in persuasion at low levels of anger, this effect disappeared under more intense anger. These findings begin to paint a picture of anger having the potential of a meaningful persuasive influence, though only under the proper conditions.

Focusing on different persuasive outcomes, anger demonstrated a stronger effect on behavioral outcomes compared to attitudes and behavioral intent. Although intriguing, these results should be considered with caution, given most of the relevant studies (84.6\%) examined effects in the context of negotiation, which differs substantially from other persuasive contexts in both the anger induction methods used (i.e., direct interpersonal interaction vs. mediated or recall-based) and the unique nature of this decision-making task (making concessions with other people's money vs. actions regarding value-laden topics such as health and politics). With these variations in mind, the results nonetheless point to the characteristic of anger as facilitating immediate action, possibly bypassing attitudinal change. Although further research is needed to examine this assertion, this speculation is supported by some evidence that suggests that at times, attitudinal change is not a prerequisite for behavioral change. For instance, Nabi and Myrick (2018) recently found that though fear and hope did not associate with attitudes towards sun safety behavior, they did associate with actual behaviors, suggesting that one does not need to 
like a behavior to enact it. A similar process may be at play with anger. That is, anger may motivate people to perform otherwise unappealing behaviors, without developing an a priori favorable attitude toward the behavior.

\section{Theory Evaluation and Theoretical Implications}

The current meta-analysis provides an opportunity to examine the validity of the theoretical frameworks that guided this investigation as well as the more or less promising directions for future research and practice in this domain. Regarding theory, it is clear that the theoretical perspectives are often more complementary than competing. The CFM is the most elaborated of the four theories, not only because it was designed to apply to different emotions, but also because it encompasses multiple aspects of message processing that are not addressed by the other models. As such, the meta-analysis largely supported all the hypotheses (H1 - H3) derived from CFM suggesting that anger is effective when it is elicited by the core message theme, coupled with a strong argument and assurance.

The AAM is intentionally narrower in its scope focusing specifically on anger and persuasion and considering fewer aspects of the persuasive process than CFM. The results regarding message relevance and efficacy are consistent with tenets of the AAM. Further, the meta-analysis’ significant findings concerning anger intensity are particularly intriguing as they illuminate the importance of further investigation of AAM. Conversely, ambient anger, which lies at the core of the ATF, evidenced a negative effect on persuasive outcomes. This finding echoes Kim and Cameron's (2011) argument that ATF is not as fruitful theoretical approach in the context of strategic communication. Further strategic communication research is needed to gain more insight into whether ambient anger can be effectively channeled to mobilize for action in a different domain (e.g., a political ad following an anger-provoking movie about an unrelated 
injustice).

Taken together, the findings from this meta-analysis suggest that to gain a more complete understanding of anger and persuasion, it is helpful to consider the CFM and AAM in combination. Specifically, self- and response-efficacy identified within the AAM fit within the broader notion of reassurance theorized in the CFM. Although efficacy constructs have received great attention and support in other contexts, fear appeals in particular, considering other ways in which messages may offer reassuring cues (e.g., through source or context cues) would be a very useful direction for future research. Similarly, though the CFM makes some propositions concerning anger intensity, the AAM emphasizes intensity as an important moderator of persuasive processes articulating specific interactive effects that were also empirically supported in the current meta-analysis. Hence, greater focus on anger intensity - both across messages and over the course of message exposure -- would be useful.

\section{Implications for Message Design}

Anger arousal in the context of persuasive messaging has been controversial due to its potential to backfire. Thus, if anger is to be harnessed for persuasive benefit, it is of paramount importance to identify recipes for effective anger appeal implementation. According to the results of this meta-analysis, the experience of anger, combined with specific message characteristics, produces a cascade of cognitive responses that ultimately can lead to either message acceptance or rejection. When irrelevant to the message, anger is more likely to result in message rejection, presumably because the message is processed more heuristically, with anger interpreted as a negative emotional peripheral cue that can taint the perception of the message and its source.

Conversely, the successful use of anger appeals arguably lies in motivating and enabling 
message consumers to process messages carefully, offering information that will compel the recipients to accept the course of action advocated in the message. To this end, a successful persuasion attempt begins with proper anger elicitation, specifically appropriate relevancy and intensity. That is, to motivate engagement with the persuasive message, anger must be deemed relevant to the issue. As argued in the context of the CFM (Nabi, 1999, 2002), anger should be elicited by the core-emotional theme in the message rather than by unrelated factors that do not motivate message-engagement. Further, if message-evoked anger is too intense, message recipients' ability to engage thoughtfully with the message is likely compromised (Loewenstein \& Lerner, 2003), which undermines persuasive potential. Thus, lower to moderate levels of anger may be more suitable for effective persuasion.

If individuals experience moderate levels of topic-relevant anger, they are presumed to engage in central message processing, in concert with findings from dual-processing models and the CFM. At this point, the strength of the argument is critical for determining the persuasive outcomes. As individuals critically scrutinize the message, weak arguments will lead to message rejection. Hence, angry individuals are more likely to adopt attitudes consistent with the message’s recommendation when they are exposed to strong, versus, weak arguments (Albarracín \& Kumkale, 2003).

Finally, to increase the likelihood of success, the message should offer a constructive outlet for aroused anger. The present meta-analysis focused on self and/or response-efficacy information as two forms of reassurances that channel the media consumers' anger into desired attitude formation and, ideally in turn, action. Conceivably, there are alternative forms of possible reassurances beyond efficacy that could similarly mobilize action (e.g., normative cues) that remain open for exploration. 


\section{Directions for Future Research and Study Limitations}

The meta-analytic approach offers a powerful tool for synthesizing existing research, but it is also confined to the research available in a given area. As such, this research highlights the gaps in the existing literature and points to particularly fruitful directions in need of further exploration. Specifically, three understudied areas of research are addressed below.

First, it has been suggested that an anger appeal is particularly effective when the message is pro-attitudinal. That is, an anger appeal can be used to mobilize individuals to act on an already held position more readily than it can be used to reach audiences prone to disagreement as the latter's anger is more likely directed, counterproductively, toward the message’s source (Turner, 2012). Unfortunately, research on anger and persuasion typically uses persuasive messages that shape or alter, rather than reinforce, attitudes, which is less amenable to examining the mobilizing effects of anger. Further, anger and persuasion studies often use topics for which individuals are likely to have preexisting attitudes, and yet do not measure participants' baseline beliefs, thus making it impossible to deduce whether the message is, in fact, counter-attitudinal or favorable. It is thus unsurprising that this meta-analysis revealed an overall very small and non-significant effect of anger on persuasion. It is essential for further research to distinguish between attitude reinforcement and attitude change effects by measuring participants’ a-priori attitudes and matching consistent/inconsistent messages.

The second understudied area identified by the meta-analysis is the scope of persuasive outcomes included in the study. The current meta-analysis focused mainly on attitudinal outcomes, given anger and persuasion studies that focus on behavioral outcomes are scarce and limited to research on negotiation. Given the identified difference between effects of anger appeals on attitudes versus behaviors, it is important to further examine whether the effect is 
confounded by the study domain (e.g., negotiation vs. other contexts) and whether the moderators tested here for attitudinal outcomes similarly apply to behavioral outcomes. Future research is needed to unpack these processes considering the effects of anger on both attitudinal and behavioral outcomes in the same domain.

Third, moving forward, research in this area would benefit from inclusion of processing variables, such as extent of elaboration and measures of counterarguing with the message. Although initially we had hoped to code for measures of message processing and reactance, the paucity of such variables $(k=4)$ forced us to rely on argument strength as a proxy for processing depth. Ideally, future studies will include measures of elaboration and reactance, which could provide important insights to the process of anger's persuasive influence.

In sum, though the overall effect of anger on persuasion across all studies was small and non-significant, the results of the current meta-analysis should be read optimistically. When executed in line with the theory-driven propositions, anger can facilitate persuasion. We hope these findings offer not only a synthesis and re-evaluation of existing research in the area of anger and persuasion, but also provide a roadmap for further investigation of the theoretical mechanisms underlying anger’s persuasive effect. 


\section{References $^{3}$}

Albarracín, D., \& Kumkale, G. T. (2003). Affect as information in persuasion: A model of affect identification and discounting, 84(3), 453-469. doi:10.1037/0022-3514.84.3.453

Averill, J. R. (1982). Anger and aggression: An essay on emotion. New York, NY: SpringerVerlag.

Begg, C. B., \& Mazumdar, M. (1994). Operating characteristics of a rank correlation test for publication bias. Biometrics, 50, 1088-1101. doi:10.2307/2533446

Borenstein, M., Hedges, L., Higgins, J., \& Rothstein, H. (2005). Comprehensive meta-analysis version 2.0. Englewood, NJ: Biostat.

Brugge, D. (1995). Pulling up the ladder: The anti-immigrant backlash. The Public Eye, 9, 1-10.

Clore, G. L., \& Ortony, A. (2008). Appraisal theories: How cognition shapes affect into emotion. In M. Lewis, J. Haviland-Jones, \& L. F. Barrett (Eds.), Handbook of emotion (3rd ed., pp. 628-642). New York: Guilfold.

De los Santos, T., \& Nabi, R. L. (in press). To share or not to share? How emotional frames influence the sharing of online news stories. Journal of Broadcasting \& Electronic Media.

Dillard, J. P., \& Nabi, R. L. (2006). The persuasive influence of emotion in cancer prevention and detection messages. Journal of Communication, 56, 123-139. doi:10.1111/j.14602466.2006.00286.x

Dimberg, U., Thunberg, M., \& Elmehed, K. (2000). Unconscious facial reactions to emotional facial expressions. Psychological science, 11, 86-89.

Duval, S., \& Tweedie, R. (2000). A nonparametric "trim and fill "method of assessing publication bias in meta-analysis. Journal of the American Statistical Association, 95, 89- 
98.

Ekman, P. (1999). Basic emotions. In T. Dalgleish, \& T. Power (Eds.), The handbook of cognition and emotion (pp. 45-60). Sussex, UK: John Wiley and Sons, Ltd.

Goleman, D. (1995). Emotional intelligence: Why it can matter more than IQ. New York: Bantam Books.

Harmon-Jones, E., \& Allen, J. J. (1998). Anger and frontal brain activity: EEG asymmetry consistent with approach motivation despite negative affective valence. Journal of Personality and Social Psychology, 74, 1310-1316. doi:10.1037/0022-3514.74.5.1310

Harmon-Jones, E., \& Sigelman, J. (2001). State anger and prefrontal brain activity: Evidence that insult-related relative left-prefrontal activation is associated with experienced anger and aggression. Journal of Personality and Social psychology, 80, 797-803. doi:10.1037/00223514.80.5.797

Harmon-Jones, E., Harmon-Jones, C., Abramson, L., \& Peterson, C. K. (2009). PANAS positive activation is associated with anger. Emotion, 9, 183-196. doi:10.1037/a0014959

Hedges, L. V., \& Vevea, J. L. (1998). Fixed- and random-effects models in meta-analysis. Psychological Methods, 3, 486-504. doi:10.1037/1082-989X.3.4.486

Higgins, J., \& Thompson, S. G. (2002). Quantifying heterogeneity in a meta-analysis. Statistics in Medicine, 21, 1539-1558. doi:10.1002/sim .1186

Ilakkuvan, V., Turner, M. M., Cantrell, J., Hair, E., \& Vallone, D. (2017). The relationship between advertising-induced anger and self-efficacy on persuasive outcomes: A test of the Anger Activism Model using the Truth Campaign. Family \& Community Health, 40, 72-80. doi:10.1097/FCH.0000000000000126

Izard, C. E., \& Ackerman, B. P. (2000). Motivational, organizational and regulatory functions of 
discrete emotions. In M. Lewis \& J. H. Haviland-Jones (Eds.), Handbook of emotion (pp. 253-264), New York: Guilford Press.

Kemper, T. D. (1987). How many emotions are there? Wedding the social and the autonomic components. American Journal of Sociology, 93, 263-289. doi:10.1086/228745

Kim, H. J., \& Cameron, G. T. (2011). Emotions matter in crisis: The role of anger and sadness in the publics’ response to crisis news framing and corporate crisis response. Communication Research, 38, 826-855.

Kim, S. J., \& Niederdeppe, J. (2014). Emotional expressions in antismoking television advertisements: consequences of anger and sadness framing on pathways to persuasion. Journal of Health Communication, 19, 692-709. doi:10.1080/10410236.2012.755603

Kühne, R., \& Schemer, C. (2015). The emotional effects of news frames on information processing and opinion formation. Communication Research, 42, 387-407. doi:10.1177/0093650213514599

Lazarus, R. S. (1993). From psychological stress to the emotions: A history of changing outlooks. Annual Review of Psychology, 44, 1-22.

Lazarus, R. S. (1999). Stress and emotion: A new synthesis. New York: Springer.

Lee, S. T., \& Cheng, I. H. (2010). Assessing the TARES as an ethical model for antismoking ads. Journal of Health Communication, 15, 55-75. doi:10.1080/10810730903460542

Lerner, J. S., \& Tiedens, L. Z. (2006). Portrait of the angry decision maker: How appraisal tendencies shape anger's influence on cognition. Journal of Behavioral Decision Making, 19, 115-137. doi:10.1002/bdm.515

Lewis, M., Sullivan, M. W., Ramsey, D. S., \& Alessandri, S. M. (1992). Individual differences in anger and sad expressions during extinction: Antecedents and consequences. Infant 
Behavior and Development, 15, 443-452. doi:10.1016/0163-6383(92)80012-J

Loewenstein, G., \& Lerner, J. S. (2003). The role of affect in decision making. In R. Davidson, K. Scherer, \& H. Goldsmith (Eds.), Handbook of affective science (pp. 619-642). New York, NY: Oxford University Press.

MacKuen, M., Wolak, J., Keele, L., \&Marcus, G. E. (2010). Civic engagements: Resolute partisanship or reflective deliberation. American Journal of Political Science, 54, 440-458. doi:10.1111/j.1540-5907.2010.00440.x.

Marcus, G. E. (2013). The Theory of Affective Intelligence and Liberal Politics. In N. Demertzis (Ed.), Emotions in politics: The affect dimension in political tension (pp. 17-38). London: Plagrave Macmillan Press.

Mitchell, M. M., Brown, K. M., Morris-Villagran, M., \& Villagran, P. D. (2001). The effects of anger, sadness and happiness on persuasive message processing: A test of the negative state relief model. Communication Monographs, 68, 347-359. doi:10.1080/03637750128070

Moons, W. G., \& Mackie, D. M. (2007). Thinking straight while seeing red: The influence of anger on information processing. Personality and Social Psychology Bulletin, 33, 706-720. doi:10.1177/0146167206298566

Nabi, R. (1998). Reasoning through emotion: An explication and test of a cognitive-functional model for the effects of discrete negative emotions on information processing, attitude change, and recall. Doctoral dissertation, University of Pennsylvania. Available from ProQuest database (UMI no. 9829960).

Nabi, R. L. (1999). A cognitive-functional model for the effects of discrete negative emotions on information processing, attitude change, and recall. Communication Theory, 9, 292-320. doi:10.1111/j.1468-2885.1999.tb00172.x 
Nabi, R. (2002). Anger, fear, uncertainty, and attitudes: A test of the cognitive-functional model. Communication Monographs, 69, 204-216. doi:10.1080/03637750216541

Noar, S. M., Pierce, L. B., \& Black, H. G. (2010). Can computer-mediated interventions change theoretical mediators of safer sex? A meta-analysis. Human Communication Research, 36, 261-297.

Panksepp, J. (1994). The basics of basic emotions. In P. Ekman \& R. J. Davidson (Eds.), The nature of emotion: Fundamental questions (pp. 20-24). New York: Oxford University Press.

Petty, R. E., \& Cacioppo, J. T. (1986). Communication and persuasion: Central and peripheral routes of attitude change. New York, NY: Springer-Verlag.

Polman, E., \& Ruttan, R. L. (2012). Effects of anger, guilt, and envy on moral hypocrisy. Personality and Social Psychology Bulletin, 38, 129-139. doi:10.1177/0146167211422365

Rains, S. A. (2013). The nature of psychological reactance revisited: A meta-analytic review. Human Communication Research, 39, 47-73. doi:10.1111/j.1468-2958.2012.01443.x

Ramírez, J., \& Andreu. (2006). Aggression, and some related psychological constructs (anger, hostility, and impulsivity) Some comments from a research project. Neuroscience \& Biobehavioral Reviews, 30, 276-291. doi:10.1016/j.neubiorev.2005.04.015

Rokeach, M. (1968). Beliefs, attitudes, and values: A theory of organization and change. San Francisco, CA: Jossey-Bass.

Rottenberg, J. Ray, R.D., \& Gross, J. J. (2007). In J.A. Coan \& J. J. B. Allen, (Eds.), Emotion elicitation using films. Handbook of emotion elicitation and assessment (pp. 9-28). London: Oxford university press.

Ryan, T. J. (2012). What makes us click? Demonstrating incentives for angry discourse with 
digital-age field experiments. The Journal of Politics, 74, 1138-1152. doi:10.1017/S0022381612000540

Schieman, S. (2010). The sociological study of anger: Basic social patterns and contexts. In: M. Potegal, G. Stemmler, C. Spielberger (Eds.), International handbook of anger (pp. 329347). New York, NY: Springer.

Schwarz, N., Bless, H., \& Bohner, G. (1991). Mood and persuasion: Affective states influence the processing of persuasive communications. In M. Zanna (Ed.), Advances in experimental social psychology (Vol. 24, pp. 161-197). New York, NY: Academic Press.

Small, D. A., \& Lerner, J. S. (2008). Emotional policy: Personal sadness and anger shape judgments about a welfare case. Political Psychology, 29, 149-168. doi:10.1111/j.14679221.2008.00621.x

Tannenbaum, M. B., Hepler, J., Zimmerman, R. S., Saul, L., Jacobs, S., Wilson, K., \& Albarracín, D. (2015). Appealing to fear: A meta-analysis of fear appeal effectiveness and theories. Psychological Bulletin, 141, 1178-1204. doi:10.1037/a0039729

Tomkins, S. S. (1962). Affect/imagery/consciousness: Vol 1. The positive affects. New York, NY: Springer.

Turner, M. M. (2007). Using emotion in risk communication: The anger activism model. Public Relations Review, 33, 114-119. doi:10.1016/j.pubrev.2006.11.013

Turner, M.M. (2012). Using emotional appeals in health messages. In: Cho, H. (Ed.), Health communication message design: Theory and practice (pp. 59-71). Los Angeles, CA: Sage. Valentino, N. A., Brader, T., Groenendyk, E. W., Gregorowicz, K., \& Hutchings, W. L. (2011). Election night's alright for fighting: The role of emotions in political participation. Journal of Politics, 73, 156-170. 
Van Dijk, E., Van Kleef, G. A., Steinel, W., \& Van Beest, I. (2008). A social functional approach to emotions in bargaining: When communicating anger pays and when it backfires. Journal of Personality and Social Psychology, 94, 600-614. doi:10.1037/00223514.94.4.600

Villalobos, J. D., \& Sirin, C. V. (2017). The relevance of emotions in presidential public appeals: Anger's conditional effect on perceived risk and support for military interventions. Presidential Studies Quarterly, 47, 146-168. doi:10.1111/psq.12349

Walter, N., Cody, M. J., Xu, L. Z., \& Murphy, S. T. (in press). A Priest, a Rabbi, and a Minister Walk into a Bar: A Meta-Analysis of Humor Effects on Persuasion. Human Communication Research. doi:10.1093/hcr/hqy005

Yip, J. A., \& Schweitzer, M. E. (2016). Mad and misleading: Incidental anger promotes deception. Organizational Behavior and Human Decision Processes, 137, 207-217. doi: $10.2139 /$ ssrn.2478692

Zuwerink, J., \& Devine, P. G. (1996). Attitude importance and resistance to persuasion: It's not just the thought that counts. Journal of Personality and Social Psychology, 70, 931-944. doi:10.1037/0022-3514.70.5.931 


\section{Footnotes}

${ }^{1}$ Studies employed different manipulations to elicit anger. The moderation analysis did not reveal significant difference $[Q(3)=2.70, p=.44]$, between the use of anger-provoking video stimuli ( $r=.39,95 \%$ CI [.19, .57], $p=.001, k=15)$, exposure to imagery of angry facial expressions ( $r=.52,95 \%$ CI $[.34, .66], p=.001, k=1)$, angry social interactions $(r=.60,95 \%$ CI [.35, .77], $p=.001, k=3)$, and recall of emotional memories $(r=.41,95 \%$ CI $[.25, .56], p=$ $.001, k=8)$.

2 The topic of the study was not a significant moderator $Q(2)=4.86, p=.12$; however, the most desirable effects were recorded in the context of education $(r=.05,95 \%$ CI $[-.09, .18], p=$ $.49)$, followed by politics ( $r=.02$, 95\% CI [-.10, .14], $p=.71)$, and health $(r=-.01,95 \%$ CI [$.20, .19], p=.94)$.

${ }^{3}$ See appendix D for all references of studies included in the meta-analysis. 


\begin{tabular}{lccccc}
\hline & & \multicolumn{2}{c}{ Heterogeneity statistics } & \multicolumn{2}{c}{ Random effects } \\
\cline { 3 - 6 } & $k$ & $I^{2}$ & $Q$ & $r$ & $95 \%$ CI \\
\hline Overall & 55 & $84.50 \%$ & $354.77^{* *}$ & .04 & {$[-.04, .10]$} \\
Attitudes & 36 & $77.55 \%$ & $155.92^{* * *}$ & -.03 & {$[-.09, .03]$} \\
Intent & 17 & $48.67 \%$ & 23.38 & .06 & {$[-.02, .14]$} \\
Behavior & 13 & $51.13 \%$ & 80.32 & $.15^{*}$ & {$[.01, .28]$} \\
\hline
\end{tabular}

Note. ${ }^{*} p<.05, * p<.01, * * * p<.001$.

Table 1. Number of studies and heterogeneity statistics for the overall random effects of anger on persuasion-related outcomes

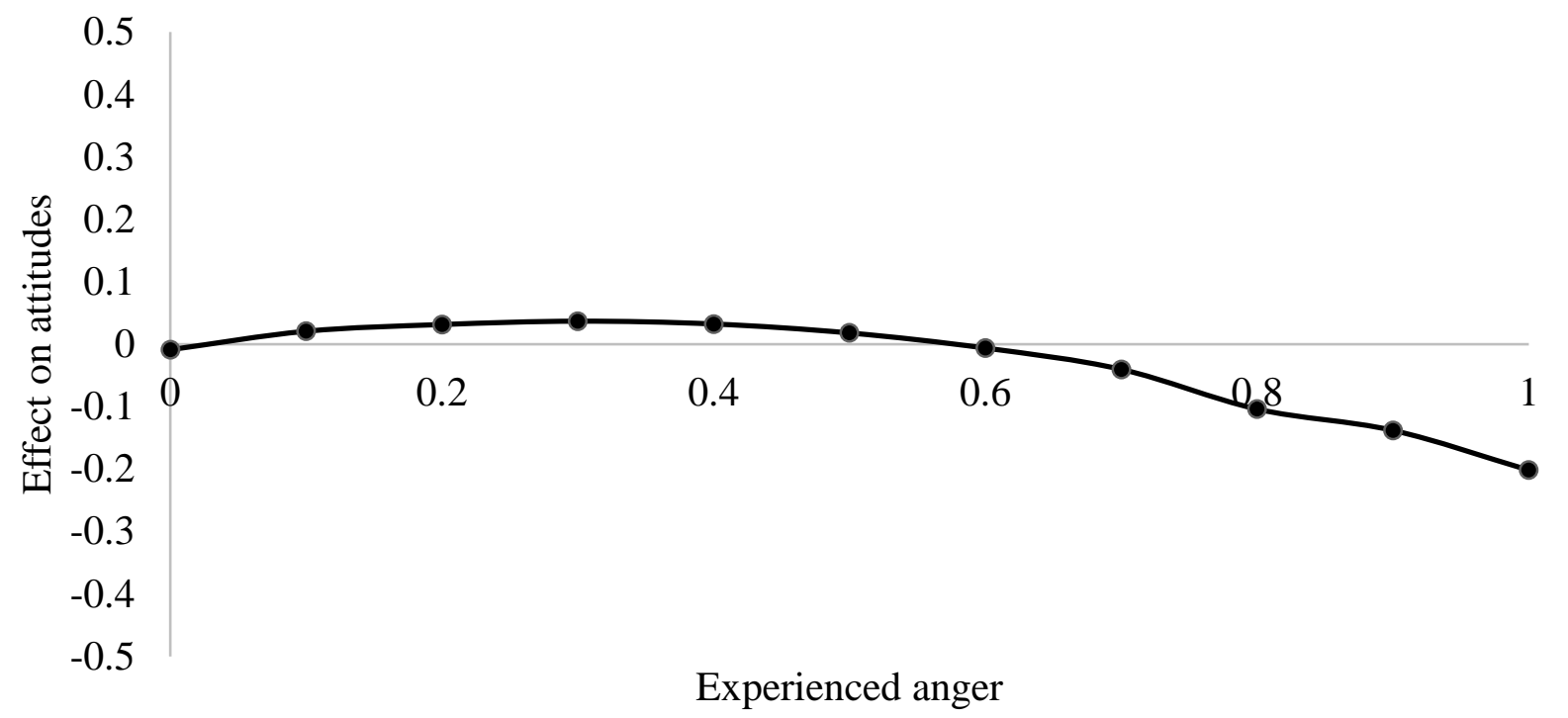

Figure 1. The plotted regression line for the prediction of attitudes by experienced anger 


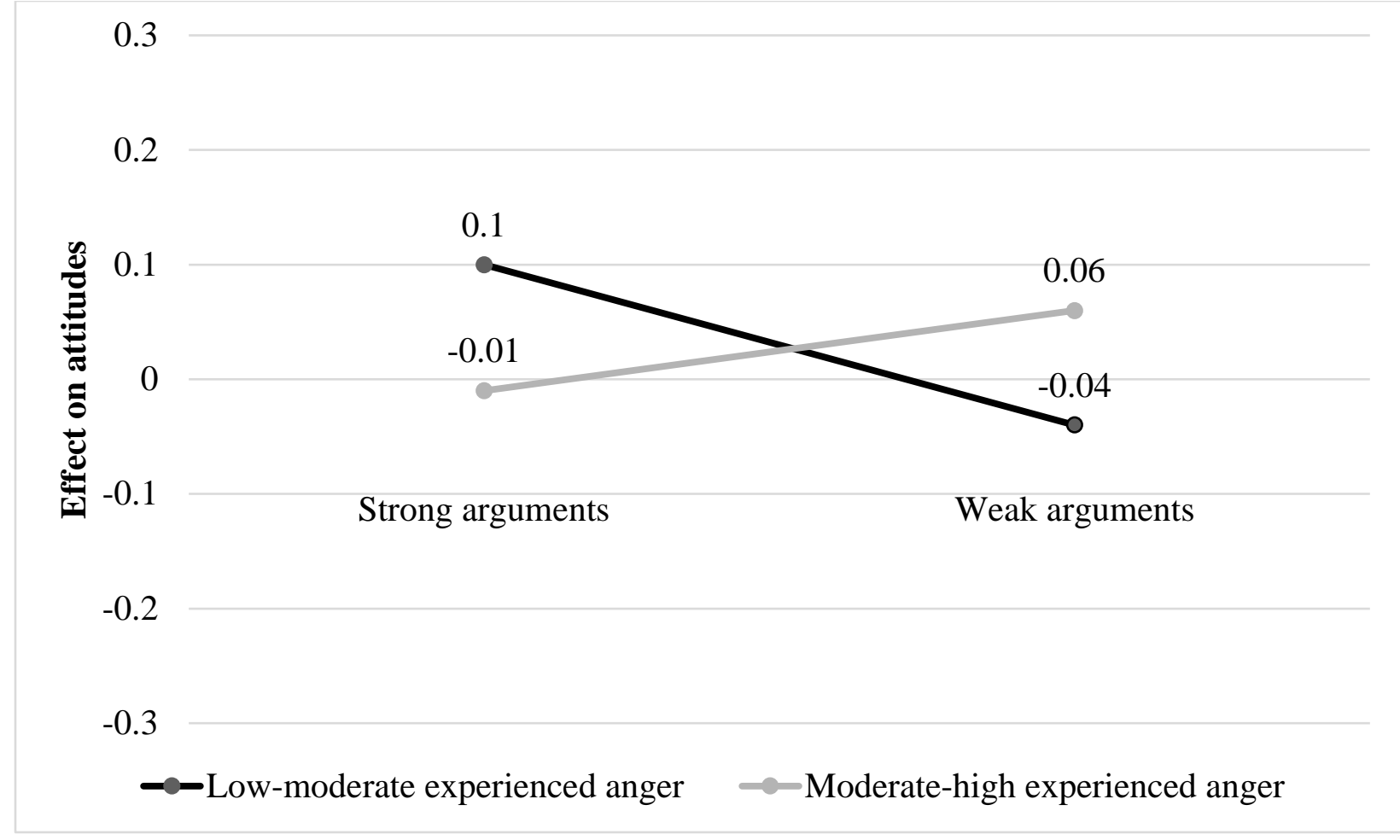

Figure 2. The interaction effect on attitudes by experienced anger and argument strength 